\title{
Use of optimized centroid and weighted centroid algorithms to locate a partial discharge source by using received signal strength.
}

KHAN, U.F., JANJUA, G.M.W., AYUB, A., ILYAS, M.A., SHAHZAD, K. and MOHAMED, $\mathrm{H}$.

(C) 2020 IEEE. Personal use of this material is permitted. Permission from IEEE must be obtained for all other uses, in any current or future media, including reprinting/republishing this material for advertising or promotional purposes, creating new collective works, for resale or redistribution to servers or lists, or reuse of any copyrighted component of this work in other works.. 


\section{Use of Optimized Centroid and Weighted Centroid Algorithms to Locate a Partial Discharge Source by Using Received Signal Strength}

\author{
Umar F. Khan \\ School of Engineering. \\ Robert Gordon University \\ Aberdeen, Scotland, UK \\ u.khan@rgu.ac.uk \\ Mohammad Adnan Ilyas \\ Electrical, Electronic and Computer \\ Engineering \\ Heriot-Watt University \\ Edinburgh, Scotland, UK
}

\author{
Ghalib M. W. Janjua \\ School of Engineering. \\ Robert Gordon University \\ Aberdeen, Scotland, UK \\ g.janjua@rgu.ac.uk \\ Khurram Shahzad \\ Electrical and Electronic Engineering \\ Bahria University \\ Islamabad, Pakistan
}

\author{
Ahmad Ayub \\ School of Engineering. \\ Robert Gordon University \\ Aberdeen, Scotland, UK \\ a.ayub2@rgu.ac.uk \\ Hamd Mohamed \\ School of Computing and Engineering \\ University of Huddersfield \\ Huddersfield, England, UK
}

\begin{abstract}
Received signal strength (RSS) based localization of a source is a simple but effective technique. In RSS based localization source location is estimated by converting obtained signal into distance. In this paper, centroid and weighted centroid algorithms has been utilized to locate a partial discharge (PD) source. An artificial PD signal was generated, and signals were captured using radio frequency (RF) sensors and hence the location of the source was estimated. The location of the source was estimated for three different positions. There were eight measurement sensors used and received signal was converted into $\mathrm{dBm}$ as input to the location algorithm.
\end{abstract}

Keywords - RSS; centroid; partial discharge; sensors.

\section{INTRODUCTION}

In high voltage systems (HV), $\mathrm{PD}$ is a phenomenon that partially bridges conductors. PD, if left unattended, will lead to catastrophic failures resulting in economic and human losses [1, 2]. Equipment such as switchgears, power generators and transformers and power cable are mainly prone to PD [3-5]. Generally, PD takes place due to insulation cracks that cause treeing between the insulation material and the conductors $[5,6]$.

A range of algorithms have been implemented in the past to estimate the location of PD in HV systems. These algorithms include time of arrival (TOA), time difference of arrival (TDOA), angle of arrival (AOA), use of SDR, USRP and difference of arrival $[3,7,8]$. Algorithms such as TOA, TDOA, AOA and DOA are all based on the localization of PD sources based on real PD pulses [9-11]. This brings the need for time based receiver node synchronization $[12,13]$.

For one off measurement and localization of PD, such algorithms are useful, however, for continuous monitoring, these time based algorithms are not plausible due to two main reasons $[11,14,15]$. Firstly, the requirement of nodes synchronization brings the need for expensive hardware and software solutions. Especially for synchronization, there will be a need of expensive clock. The second main reason is the scalability challenge [16]. In such algorithms, if the scope of the problem gets bigger, it will become a challenge to add additional receiving nodes in the existing system in place [17-19].

Considering these factors, the RSS based centroid and weighted centroid algorithms have been explored to locate the position of a PD source. The main advantage of using RSS is that, it works on the received energy rather than the real pulse itself [20]. The nodes synchronization does not remain an issue anymore due to lack of dependency on time constrained PD pulse itself. The second advantage is that in RSS based localization, the scalability is not an issue. Additional receiving nodes can be included with the system in place, should the scope of the problem gets bigger due to bigger coverage area.

\section{PROBLEM DESCRIPTION}

Partial discharge has been the topic for researchers in the past three decades. Power companies are fully aware of the phenomenon and have the systems in place to assess the condition of high voltage systems such as transformers. A common method to assess the conditions of HV systems by the power companies is to use a hand held device outside HV transformers every few months. Continuous monitoring of PD has not yet been deployed mainly due to techniques being less feasible to be deployed for continuous monitoring. Enhancements in the communication technology over the past two decades have led researchers to focus on the techniques that can be deployed for continuous monitoring of PD [21-24]. Based on this, existing centroid and weighted centroid algorithm are utilized to locate PD source. The main challenge while locating a PD source is that the location of the source is unknown as well as the source transmitted power.

The algorithm described in [25] uses the received signal and estimates the centroid based location of the source by using the received signal. The algorithm utilizes the path loss model equation, as shown in equation (1):

$$
P L\left(d_{0}\right)=32.44+10 n \log \left(d_{0}\right)+10 n \log (f)
$$

Where $P L\left(d_{0}\right)$ is the path loss of the radio signal transmitted at distance $d_{0}$, while $d_{0}$ is the distance of the reference node, $\mathrm{n}$ is the path loss coefficient of the environment ( 1 to 5 ), and $\mathrm{f}$ is the transmitted signal frequency.

\section{DESCRIPTION OF ALGORITHM}

Employing the log-normal distribution model which takes into consideration the path loss of the reference node, and the distance of the respective receiver nodes, the path loss model equation (1) can be expressed as: 


$$
P L(d)=P L\left(d_{0}\right)+10 n \log \left(d_{0}\right)+\vartheta
$$

Where $P L(d)$ is the radio signal path loss transmitted over the distance $\mathrm{d}, \vartheta$ is the Gaussian random variable having average of zero with a range of standard deviation.

The received signal strength (RSS) can be determined as:

$$
R S S I=P_{\text {send }}+P_{a m p}-P L(d)
$$

Where RSS is the received signal strength, $P_{\text {send }}$ is the transmitting power and $P_{a m p}$ is the antenna gain.

Having conducted measurements of the RSS for three different node locations, the actual transmitter location coordinates was recorded and then the centroid and weight centroid algorithms were applied to determine the estimated location of the transmitter for each case.

The conventional centroid algorithm does not take into consideration the receiver node placed at the centroid location. Also the accuracy of the common centroid algorithm is affected. Therefore, there was the need to develop an optimized centroid algorithm. As described in, the method used to determine the location of the unknown transmitted locations for the measurement results obtained is based on trilateration method. The three receiver location nodes $\mathrm{A}\left(\mathrm{x}_{\mathrm{A}}\right.$, $\left.\mathrm{y}_{\mathrm{A}}\right), \mathrm{B}\left(\mathrm{x}_{\mathrm{B}}, \mathrm{y}_{\mathrm{B}}\right), \mathrm{C}\left(\mathrm{x}_{\mathrm{C}}, \mathrm{y}_{\mathrm{C}}\right)$ are optimally selected based on the measurement RSS. Below are the key steps for the optimised centroid algorithm:

a) Assuming that the shadow effect in equation (2) is 0 then the distances from the receiver nodes to the unknown nodes can be determined as:

$$
d=10^{\left(\frac{R S S I}{10 n}\right)}
$$

b) Based on the computed distances in equation (4), rank the distances and select the three least distances from the unknown received nodes to the estimated transmitter location. The calculated ranked distances $d_{A}, d_{B}$ and $d_{C}$ are then used to determine the unknown transmitter location.

Using trilateration method, the distances obtained can be defined as:

$$
\begin{gathered}
d_{A}=\sqrt{\left(x-x_{A}\right)^{2}+\left(y-y_{A}\right)^{2}} \\
d_{B}=\sqrt{\left(x-x_{B}\right)^{2}+\left(y-y_{B}\right)^{2}} \\
d_{C}=\sqrt{\left(x-x_{C}\right)^{2}+\left(y-y_{C}\right)^{2}}
\end{gathered}
$$

c) The equations (5) to (7) have two unknowns, hence a combination of the equations is solved simultaneously to obtain the estimated transmitter location $\mathrm{x}_{1}, \mathrm{x}_{2}$ and $\mathrm{x}_{3}$. Also using the trilateration algorithm, the Centre of the triangle formed by the three anchor receiver nodes is obtained easily as used as the fourth estimated $\mathrm{x}_{4}$.

d) The optimized estimated location is then determined using centroid algorithm as follows:

$$
\begin{aligned}
& x=\frac{x_{1}+x_{2}+x_{3}+x_{4}}{4} \\
& y=\frac{y_{1}+y_{2}+y_{3}+y_{4}}{4}
\end{aligned}
$$

e) For more precise calculation, the weighted centroid algorithm can then be calculated as:

$$
\begin{aligned}
& x= \frac{\frac{x_{1}}{\left(d_{A}+d_{B}\right)}+\frac{x_{2}}{\left(d_{B}+d_{C}\right)}+\frac{x_{3}}{\left(d_{A}+d_{C}\right)}}{\frac{1}{\left(d_{A}+d_{B}\right)}+\frac{1}{\left(d_{B}+d_{C}\right)}+\frac{1}{\left(d_{A}+d_{C}\right)}} \\
& y=\frac{\frac{y_{1}}{\left(d_{A}+d_{B}\right)}+\frac{y_{2}}{\left(d_{B}+d_{C}\right)}+\frac{y_{3}}{\left(d_{A}+d_{C}\right)}}{\frac{1}{\left(d_{A}+d_{B}\right)}+\frac{1}{\left(d_{B}+d_{C}\right)}+\frac{1}{\left(d_{A}+d_{C}\right)}}
\end{aligned}
$$

Based on the above algorithms, the location of PD source was estimated. Flow chart of centroid and weighted centroid algorithms are shown in Figures 1 and 2 respectively.

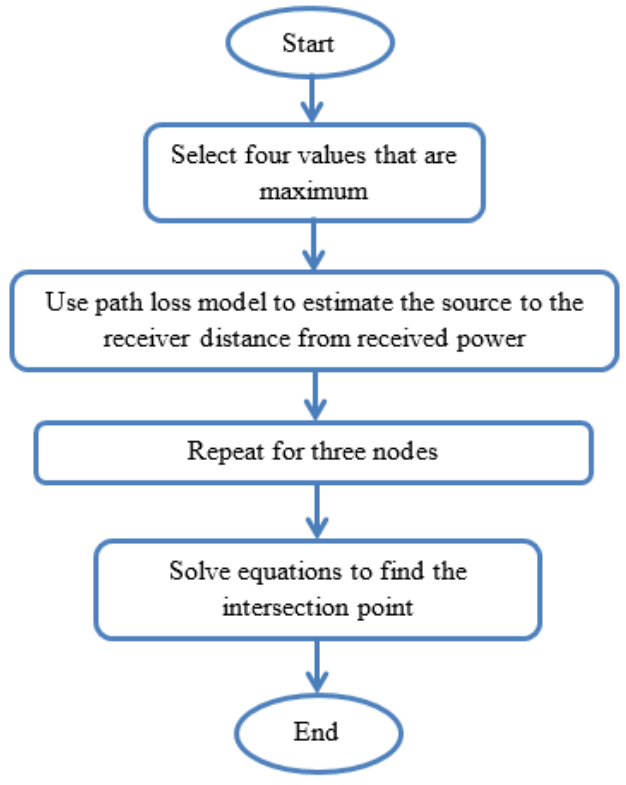

Figure 1. centroid algorithm flow chart.

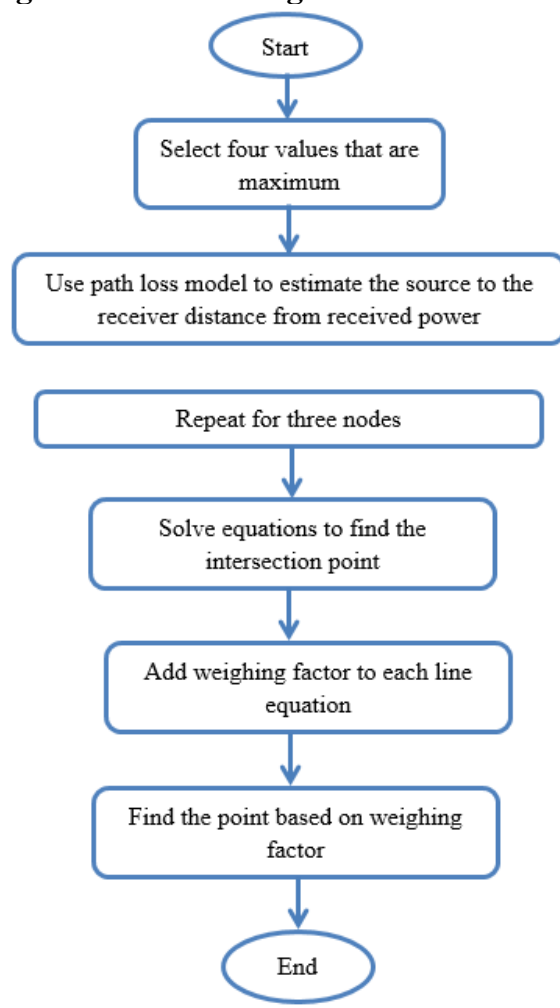

Figure 2. Weighted centroid algorithm. 


\section{EXPERIMENTAL SETUP AND RESULTS}

To check the performance of the algorithms, an artificial PD signal was generated and eight measurement sensors were used. To generate the PD signal an HVPD calibrator was used as shown in the Figure 3 below:

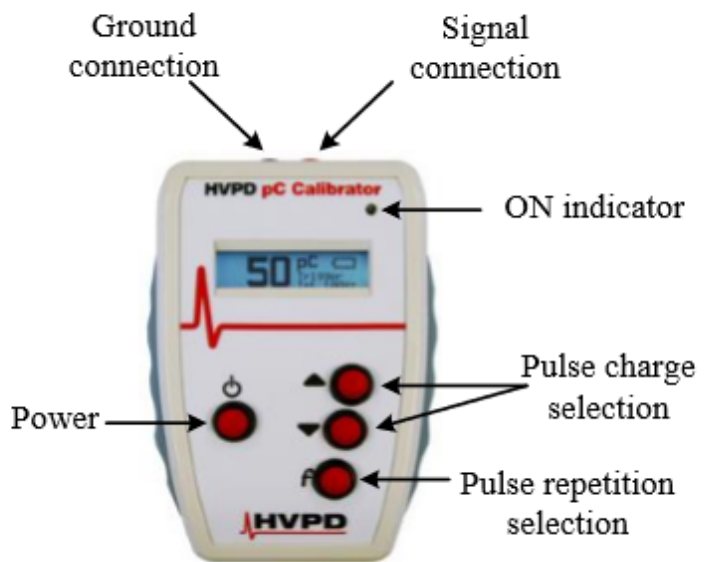

Figure 3. PD calibrator used for signal generation.

Sensors nodes were deployed for different configurations as shown in Figure 4 below:

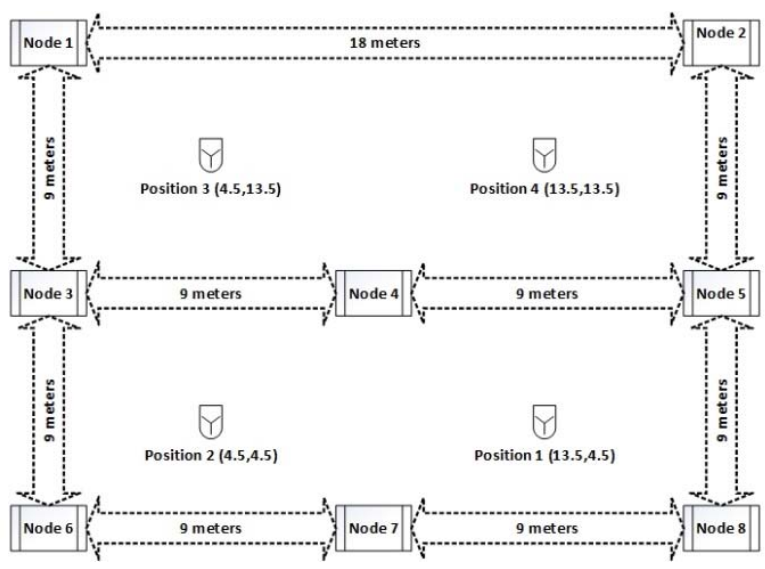

Figure 4. Sensors nodes deployment.

As shown in Figure 4, eight measurement nodes were used to perform the different measurements with source positioned at four different positions.

A PD signal of $10 \mathrm{nC}$ was generated at $100 \mathrm{~Hz}$ repetition rate. A bi-conical antenna with the frequency range from $10 \mathrm{MHz}-1 \mathrm{GHz}$ was used and the calibrator was connected to the antenna. The antenna characteristics were measured by the manufacturer to provide the antenna factor of $16.5 \mathrm{~dB} / \mathrm{m}$ at the required frequency band. To measure the signal, RF sensors were used. RF sensor had parts including RF front end, microcontroller, and signal conditioning and communication unit. Three main parts of the RF front end include the RF filters, low noise amplifier and the envelope detector. The signal conditioning unit consists of the amplifier, comparator and the mono-stable circuits. The output from signal condition unit is fed into the microcontroller unit where analogue to digital conversion of the signal is performed as well.Wireless HART is utilized to send/receive data from one node to another, it is selected among the other candidatesdue to its suitability in deployment under harsh industrial conditions. Figure 5 shows the experimental setup in a sports hall with measurement equipment used.

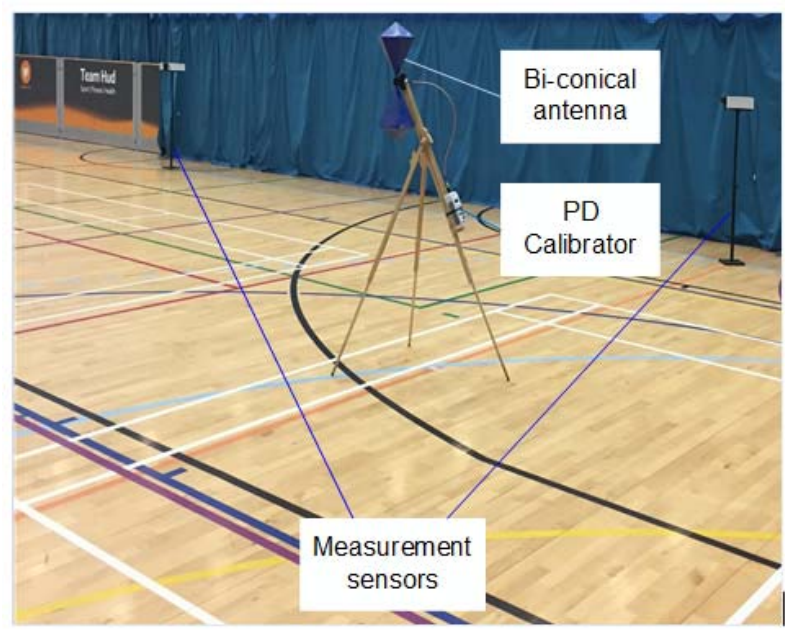

Figure 5. Experimental system

The results for all four positions are shown in Figures 6 and 7,8 and 9 respectively.

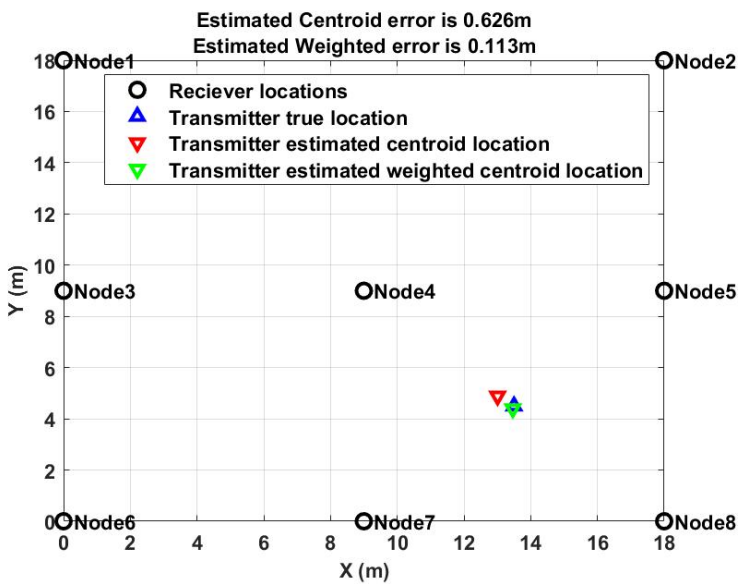

Figure 6. Position 1 estimated location.

It is evident that localisation error is below 1 meter using both algorithms.

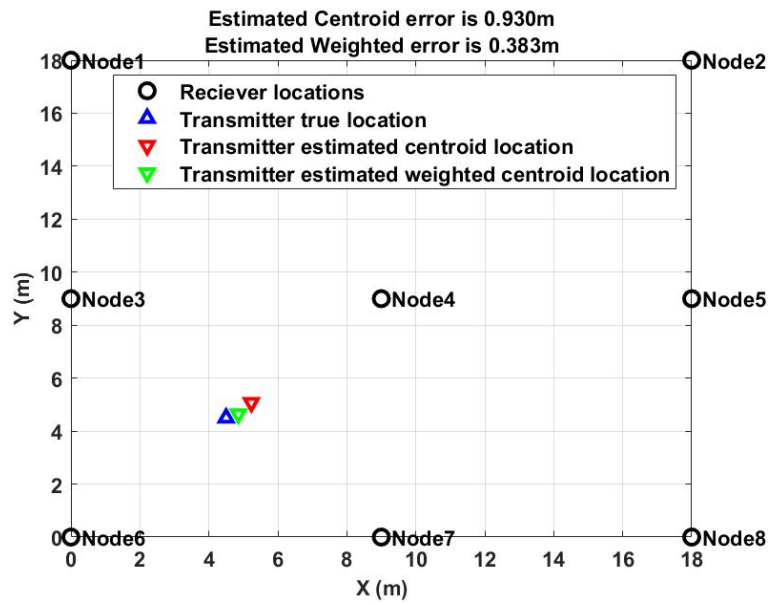

Figure 7 Position 2 estimated location 


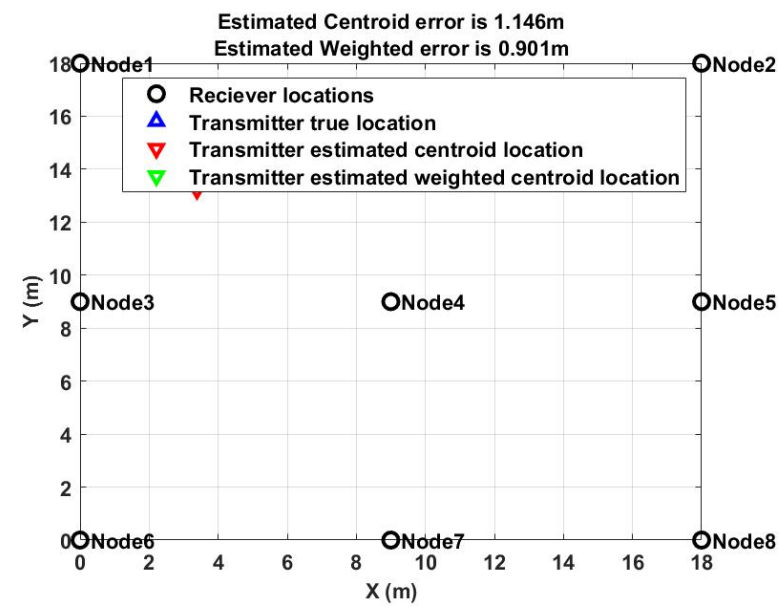

Figure 8. Position 3 estimated location.

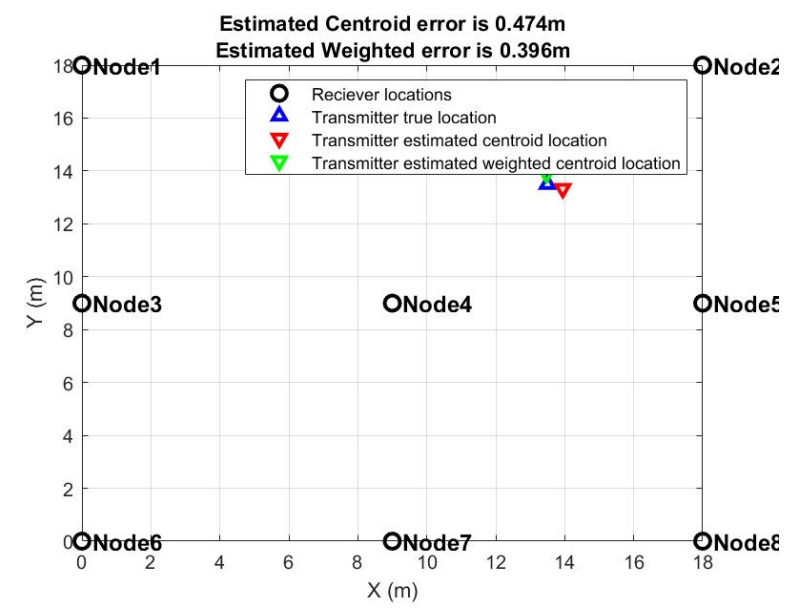

Figure 9. Position 4 estimated location.

The optimal and weighted centroid algorithm was applied on each measurement. It is important to note that the path loss exponent was set 2 for each measurement. It was observed that the centroid algorithm does not depend on the path loss exponent values therefore a default value was chosen.

\section{CONCLUSION}

RSS based localization of a PD source for four different positions was performed using centroid and weighted centroid algorithms in an indoor environment. The results show that source estimation was possible. For all four positions, source location estimation showed anrror around 1 meter or less. For all four position, wehighted centroid algorithm show the localization error remain less than 1 meter. Centroid algorithm show the localization error of 1.25 meteres for position 2 and for all other positions, the error remaine less than 1 meter. Considering the simplicity of the algorithms, the results achieved are satisfactory.

\section{REFERENCES}

[1] M. D. Judd, "Radiometric partial discharge detection," in 2008 International Conference on Condition Monitoring and Diagnosis, 2008: IEEE, pp. 1025-1030.

[2] Y. Zhang, D. Upton, A. Jaber, H. Ahmed, B. Saeed, P. Mather, P. Lazaridis, A. Mopty, C. Tachtatzis, and R. Atkinson, "Radiometric wireless sensor network monitoring of partial discharge sources in electrical substations," International Journal of Distributed Sensor Networks, vol. 11, no. 9, p. 438302, 2015.

[3] U. F. Khan, P. I. Lazaridis, H. Mohamed, R. Albarracín, Z. D. Zaharis, R. C. Atkinson, C. Tachtatzis, and I. A. Glover, "An efficient algorithm for partial discharge localization in high-voltage systems using received signal strength," Sensors, vol. 18 , no. 11, p. 4000, 2018.

[4] U. Khan, P. Lazaridis, H. Mohamed, D. Upton, K. Mistry, B. Saeed, P. Mather, R. C. Atkinson, C. Tachtatzis, and I. A. Glover, "Received signal strength intensity based localization of partial discharge in high voltage systems," in 2018 24th International Conference on Automation and Computing (ICAC), 2018: IEEE, pp. 1-5.

[5] U. Khan, P. Lazaridis, H. Mohamed, D. Upton, K. Mistry, B. Saeed, P. Mather, M. Vieira, R. Atkinson, and C. Tachtatzis, "Localization of partial discharge by using received signal strength," in 2018 2nd URSI Atlantic Radio Science Meeting (AT-RASC), 2018: IEEE, pp. 1-4.

[6] H. Mohamed, P. Lazaridis, D. Upton, U. Khan, B. Saeed, A. Jaber, Y. Zhang, P. Mather, M. Vieira, and K. Barlee, "Partial discharge detection using software defined radio," in 2016 International Conference for Students on Applied Engineering (ICSAE), 2016: IEEE, pp. 373-376.

[7] H. Mohamed, P. Lazaridis, P. Mather, C. Tachtatzis, M. Judd, R. Atkinson, and I. A. Glover, "Partial Discharge Detection and Localization: Using Software-Defined Radio," IEEE Industrial Electronics Magazine, vol. 13, no. 4, pp. 77-85, 2019.

[8] Y. Zhang, D. Upton, A. Jaber, U. Khan, B. Saeed, H. Ahmed, P. Mather, R. Atkinson, P. Lazaridis, and M. Q. Vieira, "An ultrawideband patch antenna for UHF detection of partial discharge," in $20151 s t$ URSI Atlantic Radio Science Conference (URSI AT$R A S C)$, 2015: IEEE, pp. 1-1.

[9] H. Mohamed, P. Lazaridis, D. Upton, U. Khan, K. Mistry, B. Saeed, P. Mather, M. F. Vieira, K. Barlee, and D. Atkinson, "Partial discharge localization based on received signal strength," in 2017 23rd International Conference on Automation and Computing (ICAC), 2017: IEEE, pp. 1-4.

[10] Y. Zhang, J. Neto, D. Upton, A. Jaber, U. Khan, B. Saeed, H. Ahmed, P. Mather, R. Atkinson, and J. Neto, "Radiometer monitoring system for partial discharge detection in substation," in 2015 1st URSI Atlantic Radio Science Conference (URSI ATRASC), 2015: IEEE, pp. 1-1.

[11] Y. Zhang, D. Upton, A. Jaber, H. Ahmed, U. Khan, B. Saeed, P. Mather, P. Lazaridis, R. Atkinson, and M. Q. Vieira, "Multiple source localization for partial discharge monitoring in electrical substation," in 2015 Loughborough Antennas \& Propagation Conference (LAPC), 2015: IEEE, pp. $1-4$. 
[12] H. Mohamed, P. I. Lazaridis, U. Khan, B. Saeed, K. Mistry, D. Upton, P. J. Mather, and I. A. Glover, "Partial discharge detection in smart grid using software defined radio," in 2017 25th Telecommunication Forum (TELFOR), 2017: IEEE, pp. 1-4.

[13] P. J. Moore, I. E. Portugues, and I. A. Glover, "Radiometric location of partial discharge sources on energized high-voltage plant," IEEE Transactions on Power Delivery, vol. 20, no. 3, pp. 2264-2272, 2005.

[14] A. Jaber, P. Lazaridis, M. Moradzadeh, I. Glover, Z. D. Zaharis, M. F. Vieira, M. Judd, and R. Atkinson, "Calibration of free-space radiometric partial discharge measurements," IEEE Transactions on Dielectrics and Electrical Insulation, vol. 24, no. 5, pp. 3004-3014, 2017.

[15] A. Jaber, P. Lazaridis, B. Saeed, Y. Zhang, U. Khan, D. Upton, H. Ahmed, P. Mather, M. Vieira, and R. Atkinson, "Validation of partial discharge emulator simulations using free-space radiometric measurements," in 2016 International Conference for Students on Applied Engineering (ICSAE), 2016: IEEE, pp. 475-478.

[16] D. Upton, R. Haigh, B. Saeed, U. Khan, H. Mohamed, K. Mistry, P. Mather, P. Lazaridis, F. Torres Filho, and C. Tachtatzis, "Low power highspeed folding ADC based partial discharge sensor for wireless fault detection in substations," in 2018 2nd URSI Atlantic Radio Science Meeting (ATRASC), 2018: IEEE, pp. 1-4.

[17] D. Upton, B. I. Saeed, U. Khan, A. Jaberi, H. Mohamed, K. Mistry, P. Malher, P. Lazaridis, M. Q. Vieira, and R. Atkinson, "Wireless sensor network for radiometric detection and assessment of partial discharge in HV equipment," in 2017 XXXIInd General Assembly and Scientific Symposium of the International Union of Radio Science (URSI GASS), 2017: IEEE, pp. 1-4.

[18] M. D. Judd, L. Yang, and I. B. Hunter, "Partial discharge monitoring of power transformers using UHF sensors. Part I: sensors and signal interpretation," IEEE Electrical Insulation Magazine, vol. 21, no. 2, pp. 5-14, 2005.

[19] H. Mohamed, P. Lazaridis, D. Upton, U. Khan, B. Saeed, A. Jaber, Y. Zhang, P. Mather, M. F. Vieira, and K. Barlee, "Partial discharge detection using low cost RTL-SDR model for wideband spectrum sensing," in 201623 rd International Conference on Telecommunications (ICT), 2016: IEEE, pp. 1-5.

[20] B. Saeed, D. Upton, M. Vieira, F. Torres, H. Mohamed, K. Mistry, P. Mather, P. Lazaridis, U. Khan, and C. Tachtatzis, "A supervisory system for partial discharge monitoring," in 2018 2nd URSI Atlantic Radio Science Meeting (AT-RASC), 2018: IEEE, pp. 1-4.

[21] A. Jaber, P. Lazaridis, B. Saeed, Y. Zhang, U. Khan, D. Upton, H. Ahmed, P. Mather, M. Vieira, and R. Atkinson, "Frequency spectrum analysis of radiated partial discharge signals," in Proceedings of the EUROEM, 2016.
[22] A. Jaber, P. Lazaridis, B. Saeed, Y. Zhang, U. Khan, D. Upton, H. Ahmed, P. Mather, M. d. F. Q. Vieira, and R. Atkinson, "Comparative study of Partial Discharge emulators for the calibration of Free-Space radiometric measurements," in 2016 22nd International Conference on Automation and Computing (ICAC), 2016: IEEE, pp. 313-316.

[23] A. Jaber, P. Lazaridis, Y. Zhang, B. Saeed, U. Khan, D. Upton, H. Ahmed, P. Mather, M. Vieira, and R. Atkinson, "Assessment of absolute partial discharge intensity from a free-space radiometric measurement," in 2016 URSI Asia-Pacific Radio Science Conference (URSI AP-RASC), 2016: IEEE, pp. 1011-1014.

[24] A. Jaber, P. Lazaridis, Y. Zhang, D. Upton, H. Ahmed, U. Khan, B. Saeed, P. Mather, M. F. Vieira, and R. Atkinson, "Comparison of contact measurement and free-space radiation measurement of partial discharge signals," in 2015 21st International Conference on Automation and Computing (ICAC), 2015: IEEE, pp. 1-4.

[25] H. Shi, "A new weighted centroid localization algorithm based on RSSI," in 2012 IEEE International Conference on Information and Automation, 2012: IEEE, pp. 137-141. 